\title{
Cardiomyocyte dysfunction in insulin-resistant rats: a female advantage
}

Received: 23 August 2005 / Accepted: 21 December 2005 / Published online: 16 March 2006

(C) Springer-Verlag 2006

\begin{abstract}
Aims/hypothesis: The goal of this investigation was to determine whether there are sex-related differences in the development of cardiomyocyte dysfunction in prediabetic, insulin-resistant animals. Materials and methods: Male and female rats were maintained on a high-sucrose diet for 5-11 weeks, and mechanical properties of isolated ventricular myocytes were measured by high-speed video edge detection. Several in vitro interventions were used to manipulate intracellular $\mathrm{Ca}^{2+}$ in order to determine whether altered $\mathrm{Ca}^{2+}$ availability contributes to the cardiomyocyte dysfunction. Results: Myocyte shortening and relengthening were significantly slower in sucrose-fed (insulin-resistant) males than in starch-fed (normal) male rats, whereas only relengthening was slower in sucrose-fed females when compared with normal females. Areas under the contraction and relaxation phases for sucrose-fed males were also significantly larger than in diet-matched females, and the slowed cardiomyocyte mechanics appeared earlier in males (7 vs 10 weeks). Prolonged relaxation was ameliorated in myocytes from sucrose-fed female rats by all interventions (i.e. $10^{-8} \mathrm{~mol} / \mathrm{l}$ isoprenaline, elevated extracellular $\mathrm{Ca}^{2+}$, and higher rates of stimulation). Twice as much extracellular $\mathrm{Ca}^{2+}(4 \mathrm{mmol} / \mathrm{l})$ was required to restore normal time courses of contraction and relaxation in sucrose-fed males than in females, and mechanical responses to higher
\end{abstract}

\section{L. Schwanke}

College of Arts and Sciences,

University of Maine at Farmington,

Farmington, ME, USA

\section{K. Dutta · A. J. Davidoff $(\bowtie)$}

College of Osteopathic Medicine,

University of New England,

11 Hills Beach Road,

Biddeford, ME 04005, USA

e-mail: adavidoff@une.edu

Tel.: +1-207-602-2824

Fax: +1-207-294-5931

D. A. Podolin

University of Medicine and Dentistry of New Jersey,

Stratford, NJ, USA frequency stimulation remained impaired (slower) in some myocytes from sucrose-fed male rats. Conclusions/ interpretation: These data suggest that in myocytes from insulin-resistant rats altered $\mathrm{Ca}^{2+}$ handling occurs, contributing to abnormal excitation-contraction coupling; female rats seem to have some cardioprotection during early stages in the progression towards type 2 diabetes. Females show delayed onset and milder abnormalities in metabolic status and cardiomyocyte function, but with a much tighter temporal coupling of these dysfunctions.

Keywords Contraction - Insulin resistance - Relaxation · High sucrose feeding $\cdot$ Sex differences $\cdot$ Ventricular myocytes

Abbreviations $\quad A_{C}$ : area under the contractile phase $\cdot A_{R}$ : area under the relaxation phase $\cdot \beta$-AR: $\beta$-adrenergic receptor - CaMKII: $\mathrm{Ca}^{2+}$ calmodulin kinase II $\cdot\left[\mathrm{Ca}^{2+}\right]_{\mathrm{c}}$ : extracellular $\mathrm{Ca}^{2+}$ concentration - HOMA: homeostasis model assessment $\cdot \mathrm{Max}+\mathrm{dL} / \mathrm{dt}$ : maximum rate of contraction $\cdot \mathrm{Max}-\mathrm{dL} / \mathrm{dt}$ : maximum rate of relaxation $\cdot \mathrm{PK}$ : peak shortening amplitude $\cdot$ PS: peak fractional shortening - SERCA: sarco(endo)plasmic reticulum $\mathrm{Ca}^{2+}$ ATPase

\section{Introduction}

Cardiovascular disease is a leading cause of morbidity and mortality in type 2 diabetes mellitus [1], women showing a greater increment of risk than men [2]. Abnormal ventricular systolic and diastolic functions are reported in type 2 diabetic patients presenting without macrovascular disease or hypertension, providing indirect evidence of a diabetic cardiomyopathy [3]. Furthermore, there is considerable evidence that diastolic dysfunction occurs early in the disease process [4]. In clinical studies, detectable cardiac dysfunctions have been reported to occur as early as the glucose intolerance phase (i.e. hyperinsulinaemia and hyperglycaemia) that follows insulin resistance [5].

A number of different animal models reflect aspects of human type 2 diabetes (e.g. chemically induced, diet- 
induced or genetically predisposed strains [6]). Early stages of the disease are characterised by hyperinsulinaemia, dyslipidaemia and euglycaemia (insulin-resistant phase), and later stages of the disease also include comorbidities such as hyperglycaemia, obesity, and hypertension. Because our laboratory is interested in understanding the pathogenesis of diabetic cardiomyopathy, we chose a model that presents with relatively minor metabolic abnormalities, consistent with prediabetic insulin resistance in humans [7, 8]. A high-sucrose diet is used to induce whole-body insulin resistance without producing obesity or hypertension, thereby avoiding haemodynamic changes that can independently alter cardiac physiology [9]. This model has been well characterised in terms of whole-animal glucose metabolism and lipid levels [8], and we have described a dysfunction in isolated ventricular myocytes [10] that resembles that seen in models of early stages of type 1 diabetes; i.e. prolonged myocyte shortening and relengthening and slowed cytosolic $\mathrm{Ca}^{2+}$ removal. As previously discussed $[10,11]$, this model is particularly intriguing in that impaired myocyte mechanics appears prior to overt ventricular dysfunction in the whole heart.

In contrast to clinical investigations, a number of animal studies show that female diabetic rats may have a cardiovascular advantage. For example, hearts from male rats with streptozotocin-induced type 1 diabetes have a greater degree of dysfunction than those from diabetic females [12]. In spontaneously hypertensive rats with heart failure, males (obese and non-obese) develop more severe type 2 diabetes than females and die earlier of congestive heart failure [13]. The severity of cardiovascular disease in an insulin-resistant obese strain (JCR:LA- $c p$ ) appears worse in males than in females [14]. Thus, in animals, sex may play a role in determining the time course of pathogenesis and the severity of diabetic cardiomyopathy in models of both type 1 and type 2 diabetes, although the cellular mechanisms underlying these differences remain largely unexplored.

The present study was designed to determine whether there are sex-dependent differences in the development of a cardiomyocyte dysfunction in insulin-resistant animals that do not present with overt diabetes. In addition, since it is well established that intracellular $\mathrm{Ca}^{2+}$ homeostasis is altered in heart muscle from diabetic animals (reviewed in $[15,16])$, we also examined how myocytes from sucrosefed male and female rats handle interventions that alter intracellular $\mathrm{Ca}^{2+}$.

\section{Materials and methods}

Animals and diets

All protocols were approved by the Institutional Animal Care and Use Committee at the University of New England, and conformed to the Guide for the Care and Use of Laboratory Animals (NIH Publication No. 85-23, revised 1996). Male and female Wistar rats weighing 120
$140 \mathrm{~g}$ (Charles River Breeding Laboratories, Wilmington, MA, USA) were housed individually and put on either a high-sucrose diet ( $68 \%$ of total energy from sucrose) or a control starch diet (68\% of total energy from corn starch) formulated by Research Diets (New Brunswick, NJ, USA). The diets included all necessary constituents for growth [8] and contained $68 \%$ carbohydrate, $20 \%$ protein and $12 \%$ fat. After 5-11 weeks on diet, the animals were anaesthetised by injecting a mixture of $80 \mathrm{mg} / \mathrm{kg}$ ketamine and $12 \mathrm{mg} / \mathrm{kg}$ xylazine intraperitoneally. Blood samples were collected from anaesthetised animals (prior to cardiac excision), spun in a microcentrifuge and stored $\left(-20^{\circ} \mathrm{C}\right)$ for later analysis. Plasma glucose levels were determined by the glucose oxidase method using a Beckman glucose analyser (Fullerton, CA, USA). Plasma insulin was measured by radioimmunoassay (Linco Research, St Louis, MO, USA). An estimate of insulin resistance, the homeostasis model assessment (HOMA), was calculated by the following equation: [fasting insulin $(\mu \mathrm{U} / \mathrm{ml}) \times$ fasting glucose $(\mathrm{mmol} / \mathrm{l})] / 22.5$ [17].

\section{Measurement of myocyte shortening/relengthening}

Ventricular myocytes were isolated by collagenase digestion as previously described [10], allowed to attach to coverslips coated with laminin $(10 \mu \mathrm{g} / \mathrm{ml})$, and stored in Tyrode's buffer (see below) at $37^{\circ} \mathrm{C}$ in an incubator with $100 \%$ humidity and $5 \% \mathrm{CO}_{2}$ until used. Mechanical properties were recorded in myocytes within $6 \mathrm{~h}$ after isolation and assessed by video-edge detection (IonOptix, Milton, MA, USA) as previously described [10]. Coverslips with cells attached were placed in a perfusion bath (1 ml volume) mounted on the stage of an inverted microscope. Unless otherwise specified, the cells were superfused at a flow rate of $\sim 2 \mathrm{ml} / \mathrm{min}$ with modified Tyrode's buffer of the following composition ( $\mathrm{mmol} / \mathrm{l})$ : $131 \mathrm{NaCl}, 4 \mathrm{KCl}, 1 \mathrm{MgCl}_{2}, 1 \mathrm{CaCl}_{2}, 10$ glucose, 10 HEPES, with a $\mathrm{pH}$ of 7.4 at $33 \pm 1^{\circ} \mathrm{C}$. Unless otherwise indicated, myocytes were field-stimulated to contract at a stimulus frequency of $0.5 \mathrm{~Hz}$. Images of myocyte motion were recorded at a sampling rate of $240 \mathrm{~Hz}$. Only rodshaped myocytes with clear striations and edges were selected for recording mechanical properties. Shortening and relengthening of individual myocytes were recorded only from cells that exhibited steady-state twitches. Approximately ten steady-state twitches were recorded and averaged for each myocyte. The following indices were determined with Clampfit (Axon Instruments, Foster City, CA, USA) and used to evaluate the mechanical properties of each myocyte: resting cell length, peak shortening amplitude, times to peak shortening and relengthening (10\% above baseline to $90 \%$ below peak shortening), and the area under the shortening $\left(A_{\mathrm{C}}\right)$ and relengthening $\left(A_{\mathrm{R}}\right)$ phases (Fig. 1a). $A_{\mathrm{C}}$ and $A_{\mathrm{R}}$ were normalised to peak shortening amplitude (PK) and account for both the time course and shape of the contractile cycle. The advantages and limitations of each index used to describe myocyte mechanics have been discussed pre- 
viously [18]. For clarity, peak fractional shortening (PS) and the area indices will be used in describing the subsequent experimental manipulations.

Subsets of cells were superfused with Tyrode's buffer containing either 2 or $4 \mathrm{mmol} / 1 \mathrm{Ca}^{2+}$ or $1 \mathrm{mmol} / 1 \mathrm{Ca}^{2+}$ supplemented with isoprenaline $\left(10^{-8} \mathrm{~mol} / \mathrm{l}\right)$, and fieldstimulated at $0.5 \mathrm{~Hz}$. Other cells were only stimulated at $5 \mathrm{~Hz}$ in control buffer (described above). Another subset of cells was stimulated at each of the following frequencies: 1 , 2, 3 and $5 \mathrm{~Hz}$. The cells were allowed to establish steadystate twitches after each frequency change. Only one cell per coverslip was used for each series of stimulus frequencies.

\section{Statistical analyses}

Most of the data were statistically evaluated using a $2 \times 2$ ANOVA design, in which one factor was sex and the other diet. Each index was assessed in separate ANOVAs (Systat, Evanston, IL, USA). If the interaction term (sex $\times$ diet) reached statistical significance $(p \leq 0.05)$, a Bonferroni multiple comparison test was used to identify which groups were statistically different. The most meaningful comparisons are indicated in the figures and tables, and include differences between sexes (within each diet) and between diets (within each sex). In the experiment evaluating the effects of various stimulus frequencies on the same myocyte, an ANOVA with a repeated measure design was used.

\section{Results}

Physical and metabolic status of animals

Animals were weight-matched at the beginning of the study and fed so that body weights were similar between starch and sucrose groups within each sex. After 511 weeks on diet, male rats had a significantly greater body weight than female rats, but there were no differences due to diet within each sex. Table 1 shows data for animals on diet for 9-10 weeks. Weight-matched feeding within each sex was important because obesity itself is associated with insulin resistance and cardiac dysfunctions. In this model, sucrose feeding induces whole-body insulin resistance with basal hyperinsulinaemia and euglycaemia [10]. In order to gauge whether sucrose-fed animals used in this study exhibited metabolic values characteristic of this model, blood samples were taken in anaesthetised animals just prior to excising the heart. We found, however, that the ketamine/xylazine preparation significantly suppressed insulin secretion in all groups (data not shown). Therefore, we collected blood samples in a small cohort of animals that were anaesthetised with pentobarbital $(n=4-7$ for each group, after 9-10 weeks on diet). Sucrose-fed rats of both sexes were found to be euglycaemic, hyperinsulinaemic and modestly (but significantly) insulin-resistant, as measured by HOMA, relative to starch-fed controls (Table 1).

\section{Sex differences in baseline myocyte mechanics}

Representative twitches of myocytes from starch- and sucrose-fed male and female rats are presented in Fig. 1b and c, respectively. Larger areas describing shortening and relengthening indicate slower contraction and relaxation, respectively. Isolated ventricular myocytes from sucrosefed males had prolonged shortening and relengthening when compared with myocytes from starch-fed (control) males (Fig. 1b), whereas only relengthening was prolonged in myocytes from sucrose-fed females relative to their starch-fed controls (Fig. 1c).

The time course for the development of ventricular myocyte dysfunction differed between males (Fig. 2a) and females (Fig. 2b). The entire contractile cycle (including both contraction and relaxation) can be described by the total area under the curve normalised to PK $\left(A_{\text {Total }} / \mathrm{PK}\right)$. Animals of both sexes were maintained on diet for 511 weeks. In Fig. 2, week 5 illustrates data from animals a

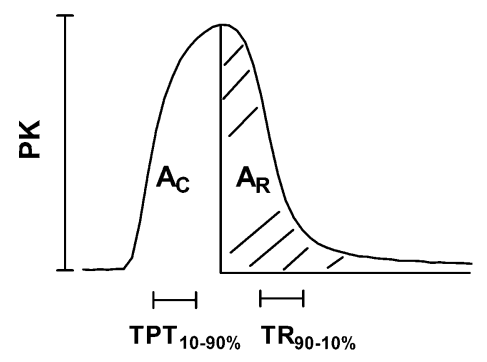

b

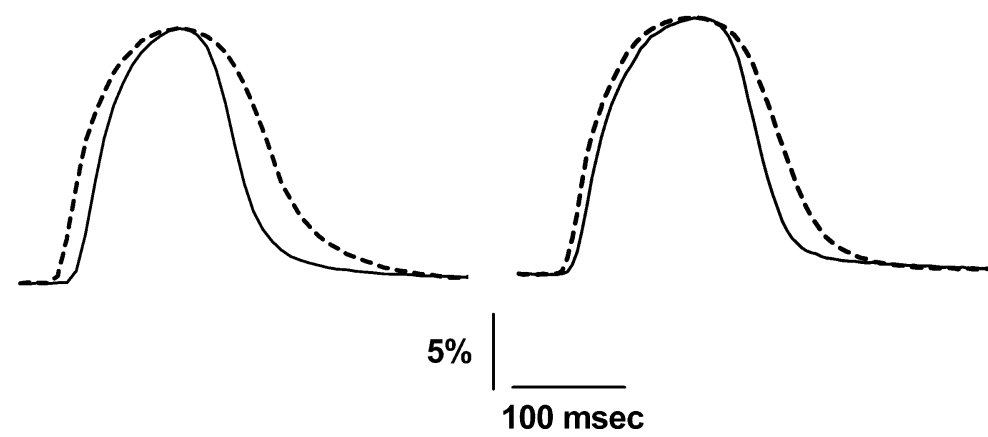

Fig. 1 a Indices used to measure shortening and relengthening of myocyte twitch. Traces depict shortening and relengthening of ventricular myocytes isolated from control starch-fed (solid lines) and high sucrose-fed (dashed lines) male (b) and female (c) rats. Myocyte twitches were recorded during electrical stimulation at $0.5 \mathrm{~Hz}$ recorded at $33^{\circ} \mathrm{C}$. Traces were chosen that best represent the means for each group of animals. Peak twitch amplitudes were normalised to each other in order to illustrate the time course of each twitch. $P K$ Peak twitch amplitude, $A_{C}$ area under the contractile phase, $A_{R}$ area under the relaxation phase, TPT time to peak twitch, $T R$ time to relaxation 
Table 1 Body weight, fasting plasma glucose, fasting insulin and insulin sensitivity (HOMA) values for rats fed either high-sucrose or control starch diet for 9-10 weeks

\begin{tabular}{lllcl}
\hline Group & Body weight $(\mathrm{g})$ & Glucose $(\mathrm{mmol} / \mathrm{l})$ & Insulin $(\mu \mathrm{U} / \mathrm{ml})$ & HOMA \\
\hline Male, starch & $548 \pm 19$ & $6.92 \pm 0.36$ & $92 \pm 20$ & $29.1 \pm 7.0$ \\
Male, sucrose & $532 \pm 20$ & $7.07 \pm 0.23$ & $147 \pm 16^{\mathrm{a}}$ & $45.3 \pm 4.9^{\mathrm{a}}$ \\
Female, starch & $288 \pm 17^{\mathrm{b}}$ & $7.01 \pm 0.11$ & $78 \pm 12$ & $24.3 \pm 3.5$ \\
Female, sucrose & $294 \pm 13^{\mathrm{b}}$ & $7.17 \pm 0.18$ & $109 \pm 20^{\mathrm{a}}$ & $34.4 \pm 5.9^{\mathrm{a}}$ \\
\hline
\end{tabular}

Data are mean \pm SEM for 4-9 animals per diet per sex

${ }^{a}$ Significantly different from starch-fed animals

${ }^{\mathrm{b}}$ Significantly different from males, independent of diet

after 5-6 weeks on diet, week 7 includes data from animals 7-8 weeks on diet, and week 10 includes data from animals on diet for 9-11 weeks. Sucrose-fed males exhibited slower myocyte mechanics by 7 weeks on diet compared with the starch-fed controls, whereas sucrose-fed females did not display mechanical abnormalities until 10 weeks on diet.

Table 2 provides a more detailed analysis of myocyte mechanical function and reveals that after 9-11 weeks on diet, shortening (or contraction) properties were slower in sucrose-fed males as described by time to peak twitch and maximum rate of shortening $(\mathrm{Max}+\mathrm{dL} / \mathrm{dt})$, as well as relengthening (or relaxation) properties as described by time to relaxation and maximum rate of relengthening
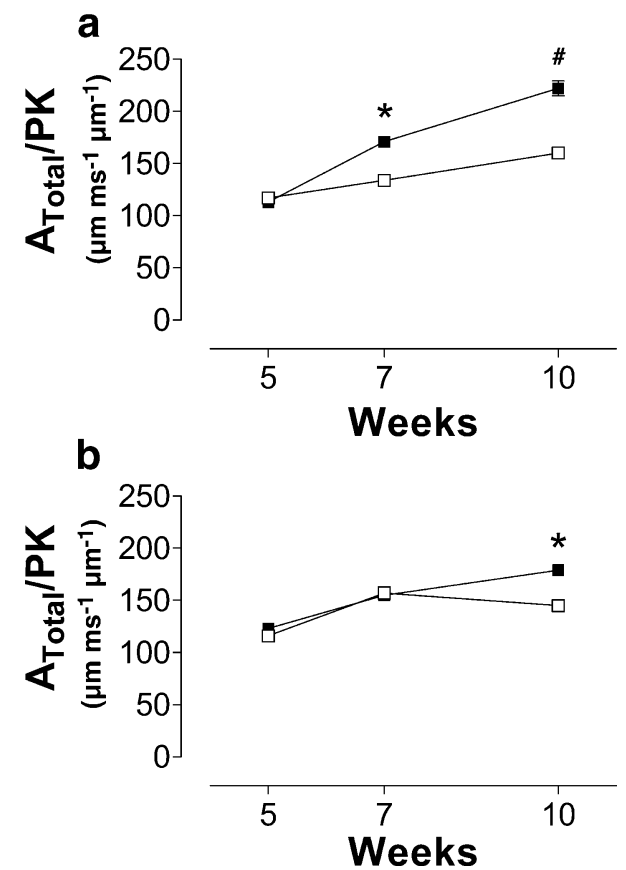

Fig. 2 Effects of diet duration on ventricular myocyte mechanics in a male and $\mathbf{b}$ female rats fed control starch (open symbol) or highsucrose (solid symbol) diets. The total area under the contraction and relaxation phases normalised to peak twitch amplitude $\left(A_{\text {Total }} / \mathrm{PK}\right)$ describes the entire contractile cycle. Data were pooled as described in Results and represent mean \pm SEM from 29-95 myocytes per sex per diet for each duration. Error bars for all data points are graphed but most are too small to show. Myocytes were isolated from 10-13 hearts per sex per diet. *Significantly different from starch-fed controls compared with the same duration of diet; " significantly different from all other groups within the same duration of diet
(Max $-\mathrm{dL} / \mathrm{dt}$ ). As mentioned above, only relengthening indices were prolonged after 9-11 weeks in sucrose-fed females, compared with their controls. These effects were also reflected in the area index for relaxation $\left(A_{\mathrm{R}} / \mathrm{PK}\right)$. Under control conditions (involving $1 \mathrm{mmol} / 1$ extracellular $\mathrm{Ca}^{2+}$ at a stimulus frequency of $0.5 \mathrm{~Hz}$ ), peak fractional shortening (PS; peak twitch amplitude normalised to resting cell length) was unaffected by diet in either sex (as illustrated in Fig 3a, with summary data in subsequent figures). Sucrose feeding did not affect the resting cell length of myocytes (Table 2); however, cells from male rats were longer than those from female rats (independent of diet). a
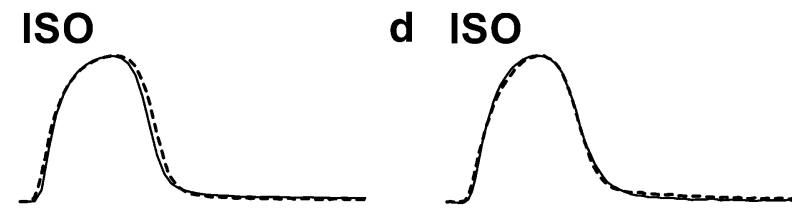

b

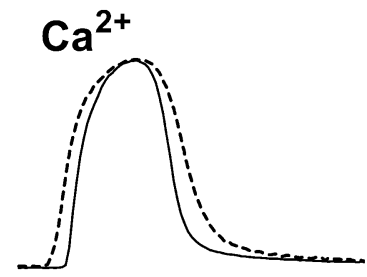

e $\mathrm{Ca}^{2+}$

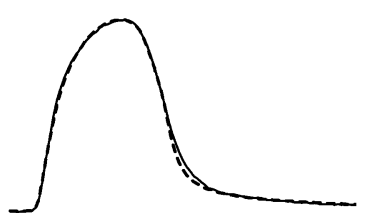

c $5 \mathrm{~Hz}$

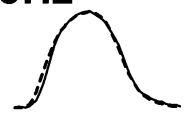

$\left.5 \%\right|_{100 \mathrm{msec}}$

f $5 \mathrm{~Hz}$

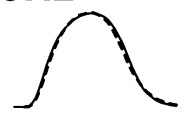

Fig. 3 Representative cardiac myocyte twitches from control starch-fed (solid lines) and sucrose-fed animals (broken lines) maintained on diet for 9-11 weeks. a-c Traces from males; d-f traces from females. The effects of $10^{-8} \mathrm{~mol} / \mathrm{l}$ isoprenaline (a, d), $2 \mathrm{mmol} / 1 \mathrm{Ca}^{2+}(\mathbf{b}, \mathbf{e})$ and $5 \mathrm{~Hz}$ stimulation $(\mathbf{c}, \mathbf{f})$ are depicted by traces that best represent average values. Summary data and statistical analyses are provided in Figs. 4 and 5. Traces are presented on the same vertical and horizontal scales 
Table 2 Effects of sex and diet on myocyte mechanics in rats after 9-11 weeks on high-sucrose or control starch diet

\begin{tabular}{lllllcll}
\hline Sex & Diet & $n$ & RCL $(\mu \mathrm{m})$ & TPT $(\mathrm{ms})$ & TR $(\mathrm{ms})$ & $\mathrm{Max}+\mathrm{dL} / \mathrm{dt}(\mathrm{mm} / \mathrm{s})$ & $\mathrm{Max}-\mathrm{dL} / \mathrm{dt}(\mathrm{mm} / \mathrm{s})$ \\
\hline Male & Starch control & 57 & $133 \pm 2$ & $59 \pm 2$ & $88 \pm 4$ & $487 \pm 27$ & $-367 \pm 20$ \\
& High sucrose & 95 & $123 \pm 3^{\mathrm{a}}$ & $79 \pm 2^{\mathrm{c}}$ & $102 \pm 5^{\mathrm{a}}$ & $308 \pm 12^{\mathrm{a}}$ & $-231 \pm 11^{\mathrm{a}}$ \\
Female & Starch control & 74 & $114 \pm 2^{\mathrm{b}}$ & $57 \pm 1$ & $74 \pm 3$ & $397 \pm 20^{\mathrm{b}}$ & $-337 \pm 20$ \\
& High sucrose & 80 & $118 \pm 2$ & $62 \pm 1$ & $113 \pm 8^{\mathrm{a}}$ & $382 \pm 19^{\mathrm{b}}$ & $-263 \pm 14^{\mathrm{a}}$ \\
\hline
\end{tabular}

Data are mean \pm SEM from five or six hearts per sex per diet

${ }^{\text {a }}$ Significantly different from starch-fed controls compared with the same sex

${ }^{\mathrm{b}}$ Significantly different from males for the same diet

${ }^{\mathrm{c}}$ Significantly different from all other groups

$R C L$ Resting cell length, TPT time to peak twitch, $T R$ time to relaxation, Max $+d L / d t$ maximum rate of contraction, $M a x-d L / d t$ maximum rate of relaxation, $n$ number of myocytes

\section{Manipulating intracellular $\mathrm{Ca}^{2+}$}

Figure 3 depicts representative myocyte twitches from both male and female animals maintained on diet for 911 weeks, and illustrates the effects of interventions used to manipulate intracellular $\mathrm{Ca}^{2+}$ levels (i.e. isoprenaline, increased extracellular $\mathrm{Ca}^{2+}$ concentration $\left(\left[\mathrm{Ca}^{2+}\right]_{0}\right)$ and $5 \mathrm{~Hz}$ stimulation). Isoprenaline (Fig. $3 \mathrm{~d}$ ), $2 \mathrm{mmol} / 1 \mathrm{Ca}^{2+}$ (Fig. 3e) and $5 \mathrm{~Hz}$ stimulation (Fig. 3f) all restored to normal the prolonged relaxation seen in sucrose-fed females. For sucrose-fed males, both isoprenaline (Fig. 3a) and $5 \mathrm{~Hz}$ stimulation (Fig. 3c) were effective in normalising myocyte contraction and relaxation. However, $2 \mathrm{mmol} / 1 \mathrm{Ca}^{2+}$ (Fig. 3b) was insufficient to restore normal function in sucrose-fed males.

Activation of the $\beta$-adrenergic receptor $(\beta-A R)$ regulates a number of mechanisms involved in $\mathrm{Ca}^{2+}$ regulation and excitation-contraction coupling (e.g. augmenting $\mathrm{Ca}^{2+}$ influx and sarcoplasmic reticulum uptake, reducing myofilament $\mathrm{Ca}^{2+}$ sensitivity). Myocytes from male and female starch- and sucrose-fed rats were superfused with $10^{-8} \mathrm{~mol} / 1$ isoprenaline and compared with recordings in control buffer (containing $1 \mathrm{mmol} / \mathrm{C} \mathrm{Ca}^{2+}$ ). As illustrated in Fig. 4a and d, isoprenaline (in the concentration used) had no effect on PS. However, it normalised both contraction $\left(A_{\mathrm{C} / \mathrm{PK}}\right)$ and relaxation $\left(A_{\mathrm{R} / \mathrm{PK}}\right)$ in myocytes from sucrose-fed males (Fig. 4e and f) and restored $A_{\mathrm{R} / \mathrm{PK}}$ in myocytes from female sucrose-fed animals (Fig. 4f).

Increasing $\left[\mathrm{Ca}^{2+}\right]_{\mathrm{o}}$ not only increases trigger $\mathrm{Ca}^{2+}$ but also increases the sarcoplasmic reticulum $\mathrm{Ca}^{2+}$ load. This manipulation produced a significant positive inotropic effect on myocytes from both starch- and sucrose-fed animals, regardless of sex (Fig. 5a, d and g). In response to $2 \mathrm{mmol} / 1 \mathrm{Ca}^{2+}, A_{\mathrm{C} / \mathrm{PK}}$ in sucrose-fed males remained abnormal (Fig. 5e), and $A_{\mathrm{R} / \mathrm{PK}}$ was restored to normal in sucrose-fed females only (Fig. 5e). In contrast to myocytes from females, in myocytes from males $4 \mathrm{mmol} / 1$ $\mathrm{Ca}^{2+}$ was required to normalise indices of mechanics (Fig. 5h and i).

The third manipulation used to alter intracellular $\mathrm{Ca}^{2+}$ was to increase stimulus frequency. We stimulated a subset of myocytes from both male and female rats at $5 \mathrm{~Hz}$ to reflect a physiologically relevant rate of contraction (in rats, typical heart rates approach 300 beats $/ \mathrm{min}$ ). Figure 6 shows that there was a negative inotropic effect (negative treppe) in both male and female myocytes (Fig. 6a,d) and that both contraction (Fig. 6e) and relaxation (Fig. 6f) were faster compared with values at the baseline rate of stimulation at $0.5 \mathrm{~Hz}$ (Fig. 6b,c). At $5 \mathrm{~Hz}$, there were no longer any significant differences between myocytes from sucrose- and starch-fed animals of either sex. These data were collected only on those myocytes that were able to achieve steady-state contractions at the higher pacing
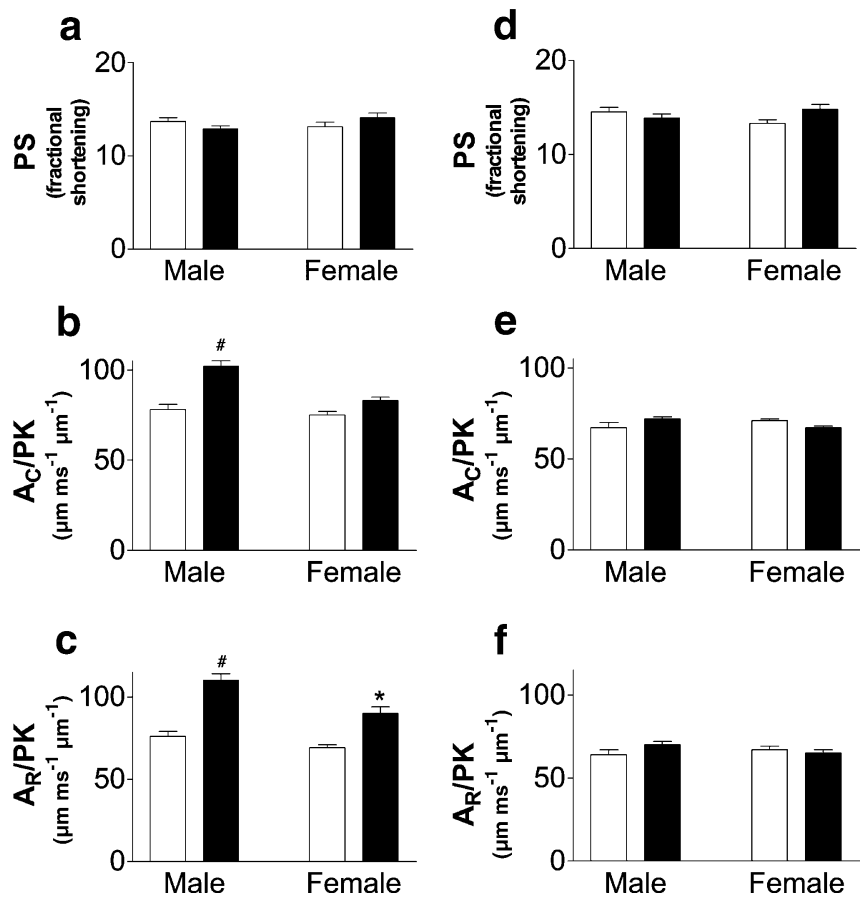

Fig. 4 Effects of $\beta$-adrenergic receptor agonist on mechanical properties of isolated myocytes from starch-fed (open bars) and sucrose-fed (solid bars) rats of both sexes after 9-11 weeks on diet. Peak fractional shortening (PS Peak twitch amplitude expressed as percentage of resting cell length) was measured in a $1 \mathrm{mmol} / \mathrm{C} \mathrm{Ca}^{2+}$ (control), or d control supplemented with $10^{-8} \mathrm{~mol} / 1$ isoprenaline (ISO). b, e Area under the contraction phase (normalised to peak twitch amplitude; $A_{\mathrm{C}} / \mathrm{PK}$ ) and $\mathbf{c}, \mathbf{f}$ area under the relaxation phase (normalised to peak twitch amplitude; $A_{\mathrm{R}} / \mathrm{PK}$ ) were measured in control $(\mathbf{b}, \mathbf{c})$ and ISO $(\mathbf{e}, \mathbf{f})$ buffers for both sexes. Myocytes were stimulated at $0.5 \mathrm{~Hz}$ and recorded at $33^{\circ} \mathrm{C}$. Data represent mean \pm SEM from 38-101 cells per diet per sex per condition, isolated from four to six hearts per diet per sex. *Significantly different from starch-fed group within sex $(p<0.05)$; ${ }^{\#}$ significantly different from all other groups 
a
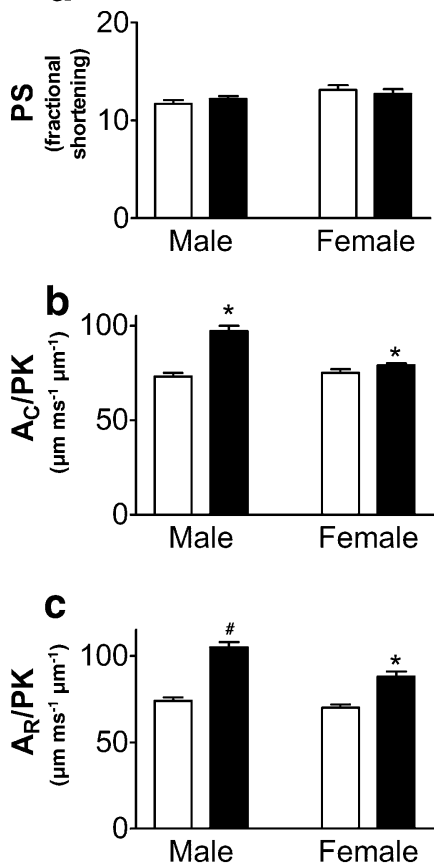

d

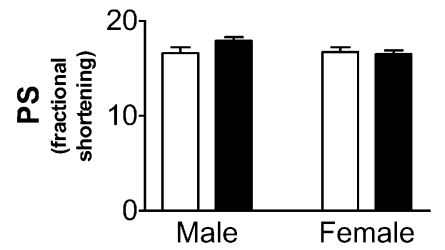

e

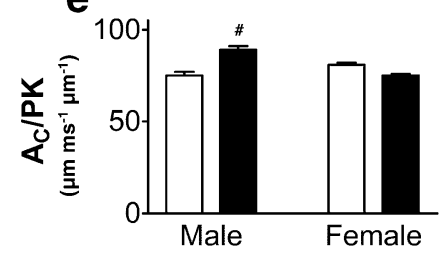

$\mathbf{f}$

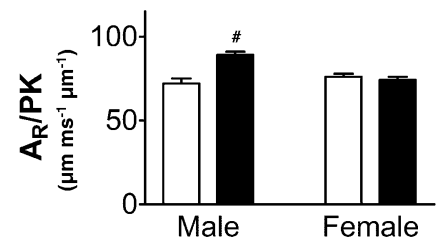

g

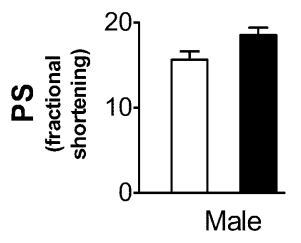

h

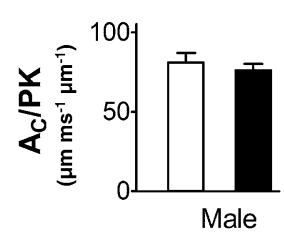

i

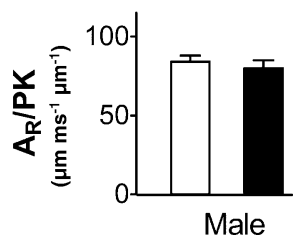

Fig. 5 Effects of changing extracellular $\left[\mathrm{Ca}^{2+}\right]$ on mechanical properties of isolated myocytes from starch-fed (open bars) and sucrose-fed (solid bars) rats of both sexes after 9-11 weeks on diet. Peak fractional shortening (PS) was measured in a $1 \mathrm{mmol} / 1 \mathrm{Ca}^{2+}$ (control) or $\mathbf{d} 2 \mathrm{mmol} / \mathrm{l} \mathrm{Ca}{ }^{2+}$ buffers in males and females, and in $4 \mathrm{mmol} / \mathrm{C} \mathrm{Ca}^{2+}$ for males only (g). $A_{\mathrm{C}} / \mathrm{PK}$ and $A_{\mathrm{R}} / \mathrm{PK}$ were also measured in $1 \mathrm{mmol} / \mathrm{l} \mathrm{Ca}^{2+}$ (control) (b, c, respectively) or $2 \mathrm{mmol} / \mathrm{l}$
$\mathrm{Ca}^{2+}$ (e, f, respectively) buffers, and in $4 \mathrm{mmol} / 1 \mathrm{Ca}^{2+}$ for males only (h, i, respectively). Myocytes were stimulated at $0.5 \mathrm{~Hz}$ and recorded at $33^{\circ} \mathrm{C}$. Data represent mean \pm SEM from 32-136 cells per diet per sex per condition, isolated from four to seven hearts per diet per sex, except for $4 \mathrm{mmol} / 1 \mathrm{Ca}^{2+}$, where $n=13-14$ cells per diet. $*$ Significantly different from starch-fed group within sex $(p<0.05)$; \# significantly different from all other groups frequency. A repeated measures experiment was also carried out with subsets of cells, each of which was stimulated over a range of frequencies (Fig. 7). In sucrosefed males, the mechanical dysfunction was apparent at all stimulus frequencies (Fig. 7a), whereas myocytes from sucrose-fed females had normal mechanics at stimulus frequencies of $1-5 \mathrm{~Hz}$ (Fig. 7b).

\section{Discussion}

The major finding of this investigation is that there are sexrelated differences in cardiomyocyte function in a prediabetic model of insulin resistance, males exhibiting more dramatic mechanical impairment and developing the dysfunction at an earlier stage than females. This cardiomyocyte dysfunction appears to be a very early manifestation of the disease process. $\beta$-Adrenergic receptor stimulation normalises function in both sexes, while other manipulations of intracellular $\mathrm{Ca}^{2+}$ (i.e. increasing $\left[\mathrm{Ca}^{2+}\right]_{\mathrm{o}}$ and stimulus frequency) were found to be more effective in restoring normal function in females than in males. Our findings suggest that the sex-related differences in the effects of sucrose feeding on cardiomyocyte excitation-contraction coupling involve changes in cytosolic $\mathrm{Ca}^{2+}$ regulation.

The cardiomyocyte dysfunction we see in the sucrosefed model appears earlier and is more severe in males, suggesting that female rats have some cardioprotection during the course of metabolic changes. At present it is impossible to say whether our findings are directly due to sex-related differences in the heart or some other factor (e.g. severity of metabolic changes associated with the high-sucrose diet). Studies with this model show that strain, age, length of time and type of diet also affect metabolic and functional outcomes $[9,19,20]$. A thorough characterisation of the time course of metabolic changes that occur in sucrose-fed male Wistar rats was carried out by Pagliassotti and colleagues [7, 21, 22] using glucose challenges and hyperinsulinaemic/euglycaemic clamp protocols in unanaesthetised animals. In a separate study, they found that sucrose-fed females were not insulin-resistant after 8 weeks and exhibited neither elevated triglycerides nor hyperinsulinaemia [23]. In the present study, we show that with an additional 1-3 weeks on diet, sucrose-fed females exhibit metabolic changes consistent with sucrosefed males (Table 1) and their ventricular myocytes have impaired relaxation, although not as severe as in males (Table 2 and Figs. 4c, 5c and 6c). The severity and duration of insulin resistance have been shown to correlate with cardiomyocyte dysfunction. For example, interventions that alter metabolism (i.e. metformin or fibrate treatment, and voluntary exercise) prevent both insulin resistance and abnormal myocyte mechanics in sucrose-fed male rats [10, 24]. This myocyte dysfunction is also completely (or 

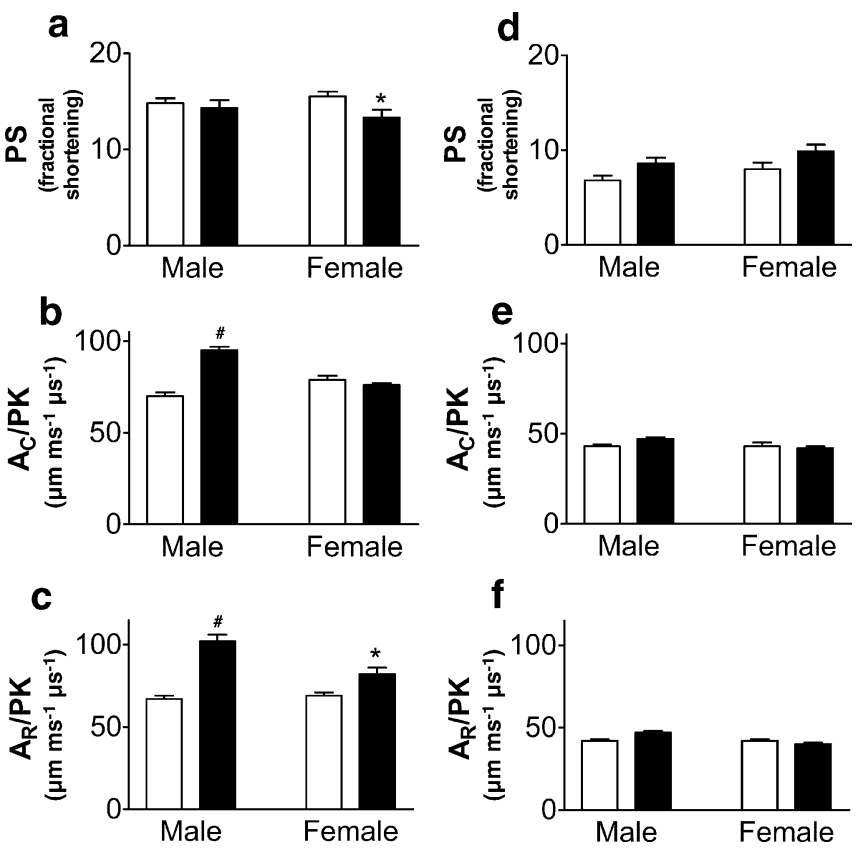

Fig. 6 Effects of increased stimulus frequency on mechanical properties of isolated myocytes from starch-fed (open bars) and sucrose-fed (solid bars) animals of both sexes after 9-11 weeks on diet. a-c Peak fractional shortening (PS), $A_{\mathrm{C}} / \mathrm{PK}$ and $A_{\mathrm{R}} / \mathrm{PK}$ respectively were recorded in normal buffer while myocytes were stimulated to contract at $0.5 \mathrm{~Hz}$ (control stimulus frequency). d-f Analysis of the same while myocytes were stimulated at $5 \mathrm{~Hz}$. Data represent mean \pm SEM from $23-42$ cells per diet per sex per frequency, isolated from five to seven hearts per diet per sex. * Significantly different from starch-fed group within sex; \# significantly different from all other groups

partially) reversible when treatments begin after the onset of insulin resistance [24].

Cardioprotection in females is evident in a number of rodent models of disease (e.g. chronic volume overload [25], remodelling after myocardial infarction [26], and recovery from ischaemia/reperfusion [27]). Data from animal models of diabetes also indicate that a female advantage persists with respect to cardiovascular disease. For example, streptozotocin-induced diabetes depresses the rate of ventricular pressure development and prolongs the contraction and relaxation phases to a greater degree in papillary muscles from males than in those from females $[12,28]$. Also, in type 1 diabetic rats, the ventricular myocyte action potential is prolonged, corresponding to a decline in peak potassium currents in males but not in females $[29,30]$. Similar attenuation of peak potassium currents in males but not females is seen in ventricular myocytes from the $d b / d b$ mouse model of type 2 diabetes with obesity [31], although whole-heart function is impaired to a similar degree in both sexes [32]. In a rat model of diabetes that uses fructose feeding, males develop insulin resistance and hypertension, while females exhibit less severe metabolic changes and no hypertension [33]. In addition, ovariectomy results in hypertension and vascular dysfunctions, as seen in fructose-fed males [33], and exacerbates metabolic changes in rats receiving high doses a

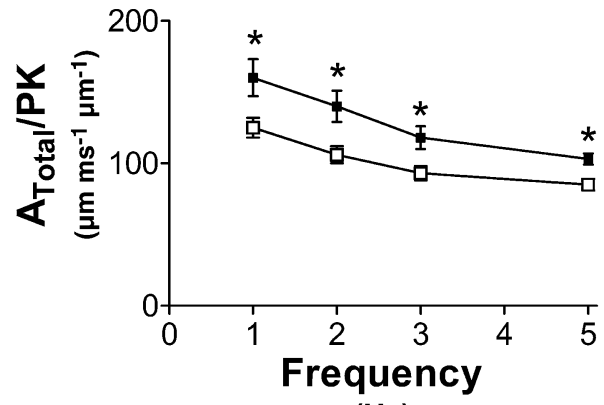

b

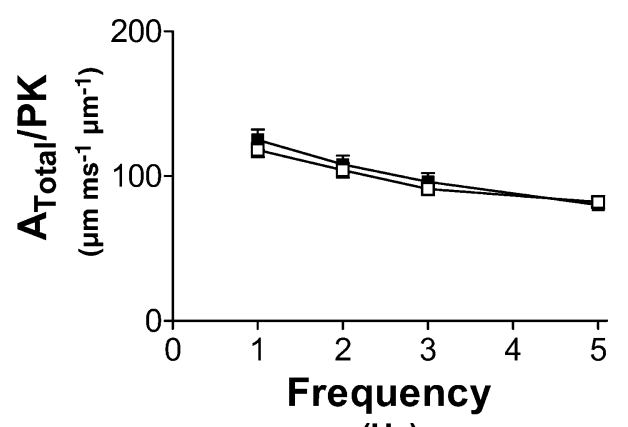

$(\mathrm{Hz})$

Fig. 7 Effects of increasing stimulus frequency on individual cardiomyocytes from starch-fed (open symbols) and sucrose-fed (solid symbols) males (a) and females (b) after 9-11 weeks on diet. $A_{\text {Total }} / \mathrm{PK}$ was recorded in the same cell at each stimulus frequency (1, 2, 3 and $5 \mathrm{~Hz})$. Data represent mean \pm SEM from 11-19 cells per diet per sex. *Significantly different from starch-fed males for a given stimulus frequency. Significant differences among frequencies are not indicated

of insulin (in place of diet-induced hyperinsulinaemia) [34].

Experimental manipulations affecting intracellular $\mathrm{Ca}^{2+}$ were undertaken based on our previous findings that $\mathrm{Ca}^{2+}$ regulation is altered in myocytes from sucrose-fed rats $[10$, $11]$, consistent with a variety of other models of cardiac dysfunction in diabetes (reviewed in $[15,16])$. Increasing extracellular $\mathrm{Ca}^{2+}$ has been shown to normalise cardiac function in streptozotocin-induced type 1 diabetic animals [35]. To test for sex-related differences in $\mathrm{Ca}^{2+}$ handling in the sucrose-fed model, we used several interventions to increase cytosolic $\mathrm{Ca}^{2+}$ (i.e. isoprenaline, increased $\left[\mathrm{Ca}^{2+}\right]_{\mathrm{o}}$ and higher frequency stimulation). Our data show that an increase in $\mathrm{Ca}^{2+}$ availability is sufficient to restore normal mechanics in myocytes from sucrose-fed females, but that this improves function to a lesser degree in males. Twice as much extracellular $\mathrm{Ca}^{2+}(4 \mathrm{mmol} / \mathrm{l})$ was needed to normalise mechanical function in myocytes from sucrosefed males compared with those from sucrose-fed females (Fig. 5), and increasing stimulus frequency was not completely effective at restoring function in male myocytes (Fig. 7). Only isoprenaline was equally effective in restoring function to cells from both male and female sucrose-fed animals (Fig. 4). Given that myocytes from males appear to be more severely affected by sucrose feeding than those from females, it is reasonable to expect 
that males cells might require higher $\left[\mathrm{Ca}^{2+}\right]_{\mathrm{o}}$ in order to compensate for the cellular defects contributing to abnormal mechanics. It is therefore interesting that the same concentration of isoprenaline was effective in normalising function in both sexes.

Stimulation of the $\beta$-AR with isoprenaline enhances the influx of trigger $\mathrm{Ca}^{2+}$, thereby affecting contraction, and facilitates relaxation through phosphorylation of troponin I and phospholamban. In our sucrose-fed model, we recently demonstrated that sarco(endo)plasmic reticulum $\mathrm{Ca}^{2+}$-ATPase (SERCA) function is depressed [11], thus providing one possible mechanism for the ability of isoprenaline to improve relaxation. Here we show that a low concentration of isoprenaline (i.e. one that does not cause positive inotropy) ameliorates cardiomyocyte dysfunction in both male and female sucrose-fed rats, although the effect is more dramatic in males (Fig. 4). This could be a function of the level of impairment induced by sucrose feeding, but could also reflect a sex-related difference in responsiveness to isoprenaline. Ventricular myocytes from males show a greater rise in $\left[\mathrm{Ca}^{2+}\right]_{\mathrm{i}}$ in response to isoprenaline [36], as well as a greater isoprenaline-mediated increase in cell shortening, L-type $\mathrm{Ca}^{2+}$ current and cyclic AMP production, associated with a two-fold greater $\beta$-AR density relative to myocytes from females [37]. In any case, the improved mechanical function we see with isoprenaline suggests that a subtle dysfunction exists in myocytes from sucrose-fed rats, because it is corrected with $\beta$-AR stimulation. This is consistent with the observation that isoprenaline stimulation also normalises defective excitation-contraction coupling in myocytes from non-failing hypertrophic hearts but not in myocytes from failing hypertrophic hearts [38].

Increasing stimulus frequency may lead to an overall accumulation of intracellular $\mathrm{Ca}^{2+}$, even though the trigger $\mathrm{Ca}^{2+}$ entering with each beat may be smaller due to a shortening of action potential duration. Accordingly, with $5 \mathrm{~Hz}$ stimulation we see a negative inotropy, accompanied by a faster rate of contraction and relaxation in myocytes from both starch- and sucrose-fed animals, a finding that is independent of sex (Fig. 6d-f). Interestingly, approximately the same proportion of myocytes from sucrose- and starch-fed females $(\sim 80 \%)$ could be driven to contract at $5 \mathrm{~Hz}$, whereas we have previously reported [11] a striking difference between myocytes from sucrose- and starch-fed males in terms of capability to be paced at $5 \mathrm{~Hz}(50 \mathrm{vs} 84 \%$, respectively). As reported in an earlier study [10], sucrose feeding does not produce uniformly adverse effects on all cardiac myocytes, such that a subset of these heart cells appears to have 'normal' function. Thus, the data presented in Fig. 6 may be biased in favour of cells that are only mildly affected by the long-term sucrose feeding. In order to better refine the question of frequency-dependent effects, a subset of cells was subjected to a range of stimulus frequencies and analysed with a repeated measures ANOVA (Fig. 7). This analysis revealed that some myocytes from sucrose-fed males retain their mechanical dysfunction over a range of frequencies, whereas female myocytes are able to respond with normal twitch dynamics at frequencies from 1 to $5 \mathrm{~Hz}$.

There is evidence to suggest that, in normal rat ventricular myocytes, upregulation of $\mathrm{Ca}^{2+} /$ calmodulindependent protein kinase II (CaMKII) activity in response to either increased stimulus frequency or $\left[\mathrm{Ca}^{2+}\right]_{\mathrm{o}}$ increases the rate of intracellular $\mathrm{Ca}^{2+}$ clearing and mechanical relaxation [39]. This mechanism of CaMKII upregulation could explain the normalisation of mechanical functions in our myocytes from sucrose-fed animals exposed to isoprenaline, increased $\left[\mathrm{Ca}^{2+}\right]_{\mathrm{o}}$ or $5 \mathrm{~Hz}$ stimulation. We have tried a CaMKII inhibitor with an in vitro model of high-glucose-induced myocardial dysfunction, and have preliminary evidence that CaMKII is required for the improvement of relaxation by high $(4 \mathrm{mmol} / \mathrm{l})\left[\mathrm{Ca}^{2+}\right]_{\mathrm{o}}$ (Dutta and Davidoff, unpublished data). In that model, as in the sucrose-fed model, impaired SERCA function has been demonstrated $[11,40]$. Whether a sex-related difference in CaMKII level or activity occurs with sucrose feeding remains to be investigated.

The sucrose-fed model provides a means of analysing changes in metabolic state and cardiac dysfunction without complications such as coronary vascular disease and obesity. Hypertension has been shown to develop in sucrose-fed rats, but only when juveniles were used [19] rather than adults [9]. While the typical atherosclerosis resistance of rats [14] might be considered a limitation of this model and may help explain some of the differences seen in experimental and clinical studies of type 2 diabetes, the ability to investigate cardiac changes without vascular complications is an important advantage of using sucrosefed rats. In a previous study we demonstrated that a number of interventions can prevent or reverse both the insulin resistance and cardiomyocyte dysfunction induced by sucrose feeding [24], thereby demonstrating the usefulness of this model in elucidating very early changes in the disease process. Here we provide evidence of sex-related differences in the development of the disease, thus adding importance to future investigations using this model. Males begin to exhibit metabolic abnormalities after just 2 weeks on diet [7], but do not develop significant myocardial dysfunction until at least 6 weeks of sucrose feeding (Fig. 2). Females, on the other hand, show delayed onset and milder abnormalities in both metabolic status and cardiomyocyte function, but with a much tighter temporal coupling of these dysfunctions (i.e. signs of insulin resistance in females begin to appear after 9-11 weeks on diet [Table 1], with a corresponding impairment of cardiomyocyte relaxation [Fig. 2]). Thus, one aspect of future studies should include a careful investigation of the linkage between sucrose-induced insulin resistance and cardiac dysfunction in males vs females. The sucrose-fed model provides an important means for studying the impact of sex on cellular changes in cardiac muscle during the early stages of development of type 2 diabetes and/or metabolic syndrome. 
Acknowledgements This research was supported by funding from the National Institutes of Health R01-HL60303 and R01-HL66895 (to A. J. Davidoff).

\section{References}

1. Zimmet P, Alberti MM, Shaw J (2001) Global and societal implications of the diabetes epidemic. Nature 414:7827-7887

2. Barrett-Connor E, Giardine EG, Gitt AK, Gudat U, Steinber HO, Tschoepe D (2004) Women and heart disease: the role of diabetes and hyperglycemia. Arch Intern Med 164:934-942

3. Zarich SW, Nesto RW (1989) Diabetic cardiomyopathy. Am Heart J 118:1000-1012

4. Raev DC (1994) Which left ventricular function is impaired earlier in the evaluation of diabetic cardiomyopathy. Diabetes Care 17:633-639

5. Celentano A, Vaccaro O, Tammaro P et al (1995) Early abnormalities of cardiac function in non-insulin-dependent diabetes mellitus and impaired glucose tolerance. Am J Cardiol 76:1173-1176

6. McNeill JH (1999) Experimental models of diabetes. CRC, Boca Raton, Florida

7. Pagliassotti MJ, Prach PA, Koppenhafer TA, Pan DA (1996) Changes in insulin action, triglycerides, and lipid composition during sucrose feeding in rats. Am J Physiol 271:R1319-R1326

8. Podolin DA, Gayles EC, Wei Y, Thresher JS, Pagliassotti MJ (1998) Menhaden oil prevents but not reverse sucrose-induced insulin resistance in rats. Am J Physiol 274:R840-R848

9. Santuré M, Pitre M, Marette A et al (2002) Induction of insulin resistance by high-sucrose feeding does not raise mean arterial blood pressure but impairs haemodynamic responses to insulin in rats. Br J Pharmacol 137:185-196

10. Dutta K, Podolin DA, Davidson MB, Davidoff AJ (2001) Cardiomyocyte dysfunction in sucrose-fed rats is associated with insulin resistance. Diabetes 50:1186-1192

11. Wold LE, Dutta K, Mason MM et al (2005) Impaired SERCA function contributes to cardiomyocyte dysfunction in insulin resistant rats. J Mol Cell Cardiol 39:297-307

12. Rodrigues B, McNeill JH (1987) Comparison of cardiac function in male and female diabetic rats. Gen Pharmacol 18:421-423

13. McCune SA, Jenkins JE, Stills HF et al (1990) Renal and heart function in the SHHF/Mcc-cp rat. In: Frontiers in Diabetes Research III. Smith Gordon, London, pp 397-401

14. Clark TA, Pierce GN (2000) Cardiovascular complications of non-insulin-dependent diabetes. The JCR:LA-cp rat. J Pharmacol Toxicol Methods 43:1-10

15. Schaffer SW, Mozaffari M (1996) Abnormal mechanical function in diabetes: relation to myocardial calcium handling. Coron Artery Dis 7:109-115

16. Pierce GN, Russell JC (1997) Regulation of intracellular $\mathrm{Ca}^{2+}$ in the heart during diabetes. Cardiovasc Res 34:41-47

17. Emoto M, Nishizawa Y, Maekawa K et al (1999) Homeostasis model assessment as a clinical index of insulin resistance in type 2 diabetic patients treated with sulfonylureas. Diabetes Care 22:818-822

18. Ren J, Davidoff AJ (1997) Diabetes rapidly induces contractile dysfunctions in isolated ventricular myocytes. Am J Physiol 272:H148-H158

19. Hulman S, Falkner B (1994) The effect of excess dietary sucrose on growth, blood pressure, and metabolism in developing Sprague-Dawley rats. Pediatr Res 36:95-101

20. Kim J-Y, Nolte LA, Hansen PA, Han D-H, Kawanaka K, Holloszy JO (1999) Insulin resistance of muscle glucose transport in male and female rats fed a high-sucrose diet. Am J Physiol 276:R665-R672
21. Pagliassotti MJ, Prach PA (1995) Quantity of sucrose alters the tissue pattern and time course of insulin resistance in young rats. Am J Physiol 269:R641-R646

22. Pagliassotti MJ, Gayles EC, Podolin DA, Wei Y, Morin CL (2000) Developmental stage modifies diet-induced peripheral insulin resistance in rats. Am J Physiol 278:R66-R73

23. Horton TJ, Gayles EC, Prach PA, Koppenhafer TA, Pagliassotti MJ (1997) Female rats do not develop sucrose-induced insulin resistance. Am J Physiol 272:R1571-R1576

24. Davidoff AJ, Mason MM, Davidson MB et al (2004) Sucroseinduced cardiomyocyte dysfunction is both preventable and reversible with clinically relevant treatments. Am J Physiol 286:E718-E724

25. Brower GL, Gardner JD, Janicki JS (2003) Gender mediated cardiac protection from adverse ventricular remodeling is abolished by ovariectomy. Mol Cell Biochem 251:89-95

26. Litwin SE, Katz SE, Litwin CM, Morgan JP, Douglas PS (1999) Gender differences in postinfarction left ventricular remodeling. Cardiology 91:173-183

27. Imahashi K, London R, Steenbergen C, Murphy E (2004) Male/ female differences in intracellular $\mathrm{Na}^{+}$regulation during ischemia/reperfusion in mouse heart. $\mathrm{J}$ Mol Cell Cardiol 37:747-753

28. Brown RA, Filipovich P, Walsh MF, Sowers JR (1996) Influence of sex, diabetes, and ethanol on intrinsic contractile performance of isolated rat myocardium. Basic Res Cardiol 91:353-360

29. Shimoni Y, Liu X-F (2003) Sex differences in the modulation of $\mathrm{K}^{+}$currents in diabetic rat cardiac myocytes. J Physiol 550:401-412

30. Shimoni Y, Liu X-F (2004) Gender differences in ANG II levels and action on multiple $\mathrm{K}^{+}$current modulation pathways in diabetic rats. Am J Physiol 287:H311-H319

31. Shimoni Y, Chuang M, Abel ED, Severson DL (2003) Genderdependent attenuation of cardiac potassium currents in type 2 diabetic $\mathrm{db} / \mathrm{db}$ mice. J Physiol 555:345-354

32. Aasum E, Hafstad AD, Severson DL, Larsen TS (2003) Agedependent changes in metabolism, contractile function, and ischemic sensitivity in hearts from $\mathrm{db} / \mathrm{db}$ mice. Diabetes $52: 434-441$

33. Galipeau DM, Verma S, McNeill JH (2002) Female rats are protected against fructose-induced changes in metabolism and blood pressure. Am J Physiol 283:H2478-H2484

34. Galipeau DM, Yao L, McNeill JH (2002) Relationship among hyperinsulinemia, insulin resistance, and hypertension is dependent on sex. Am J Physiol 283:H562-H567

35. Bielefeld DR, Pace CS, Boshell BR (1983) Altered sensitivity of chronic diabetic rat heart to calcium. Am J Physiol 245: E560-E567

36. Curl CL, Wendt IR, Kotsanas G (2001) Effects of gender on intracellular $\left[\mathrm{Ca}^{2+}\right]$ in rat cardiac myocytes. Pflugers Arch 441:709-716

37. Vizgirda VM, Wahler GM, Sondgeroth KL, Ziolo MT, Schwertz DW (2002) Mechanisms of sex differences in rat cardiac myocyte response to $\beta$-adrenergic stimulation. Am J Physiol 282:H256-H263

38. Gómez AM, Valdivia HH, Cheng H et al (1997) Defective excitation-contraction coupling in experimental cardiac hypertrophy and heart failure. Science 276:800-806

39. Bassani AR, Mattiazzi A, Bers DM (1995) CaMKII is responsible for activity-dependent acceleration of relaxation in rat ventricular myocyte. Am J Physiol 268:H703-H712

40. Dutta K, Carmody MW, Cala SE, Davidoff AJ (2002) Depressed PKA activity contributes to impaired SERCA function and is linked to the pathogenesis of glucose-induced cardiomyopathy. J Mol Cell Cardiol 34:985-996 